\title{
Short burst oxygen treatment for breathlessness in chronic obstructive airways disease
}

\author{
T W EVANS, J C WATERHOUSE, A CARTER, J F NICHOLL, P HOWARD \\ From the Academic Division of Medicine, University of Sheffield, Royal Hallamshire Hospital, Sheffield
}

ABSTRACT Most patients with chronic obstructive lung disease prescribed oxygen at home by their family doctor use it for short periods when they feel breathless. Many patients are normoxic. Nineteen patients with advanced disease and variable hypoxaemia undertook exercise until they indicated severe breathlessness on a $100 \mathrm{~mm}$ visual analogue scale. Air, $67 \%$ oxygen, and air delivered from a cylinder in an identical manner to the oxygen were randomly administered during the recovery periods. Respiratory rate, heart rate and oxygen and carbon dioxide tensions, estimated by transcutaneous electrodes $\left(\mathrm{tcPO}_{2}, \mathrm{tcPCO}_{2}\right)$, were measured throughout the exercise and recovery periods. The mean recovery time for breathlessness as judged by visual analogue score was significantly shorter when oxygen was used than during placebo or air recovery $(\mathrm{p}<0.05)$. The rates of return to baseline levels of respiratory and heart rates were not significantly affected by the gas inhaled. No placebo effect was detectable. It was not possible to select good responders from the results of prior physiological tests. In seven patients the study was repeated after an interval of between one week and a year. The consistency of observed response to oxygen was poor. Although recovery judged by visual analogue scores showed some consistency within individual patients and greater overall consistency than heart rate or respiratory rate, the direction of change in recovery judged by visual analogue score after the breathing of oxygen was variable. The change during the breathing of oxygen was towards improvement in both studies in three patients and towards deterioration in both studies in one patient, and it showed no consistent direction of change in the remaining three patients. It is difficult to determine which patients will derive substantial and reproducible benefit from short burst oxygen but their numbers are probably small. The results cast doubt on the justification for the current widespread prescription of oxygen cylinders for occasional use.

Breathlessness and reduced exercise tolerance are the major complaints of patients suffering from chronic airway obstruction. ${ }^{1}$ The conventional treatment of this condition has been with $\beta_{2}$ agonists and, in severe cases, home oxygen. ${ }^{2}$ The prescription of oxygen for such patients is, however, haphazard. Studies in Southampton and Sheffield indicate that, although oxygen is prescribed for the relief of hypoxaemia, it is taken by patients for the relief of breathlessness. ${ }^{3} 4$ As few instructions are given to patients other than from the delivering chemists and as oxygen is taken via a face mask at flow rates of 2-4 litres a minute, for

Address for reprint requests to: Dr $\mathbf{P}$ Howard, Department of Medicine, Royal Hallamshire Hospital, Sheffield S10 2JF.

Accepted 10 February 1986 a few minutes to half an hour, it can clearly be of little use in the prolonged relief of hypoxaemia. Physicians have therefore discounted the value of oxygen used in this manner, regarding improvements in dyspnoea as a placebo response to a highly emotive treatment. The measurement of subjective sensations such as breathlessness is difficult, but recently the use of the visual analogue scale of breathlessness has proved remarkably useful. ${ }^{5-8}$ Portable oxygen in patients with obstructive airways disease and severe breathlessness during exercise marginally increases distance walked but has a greater effect on relief of breathlessness. ${ }^{56}$

The present study extends the use of the visual analogue scale to measure the improvement in breathlessness claimed by most users of home oxygen after short bouts of exercise. An attempt has been made to use the visual analogue scale and certain 
Table 1 Baseline (resting) measurements for 19 patients

\begin{tabular}{lllllr}
\hline FEV/FVC (l) & $\begin{array}{l}\mathrm{PaO}_{2} \\
(\mathrm{kPa})\end{array}$ & $\begin{array}{l}\mathrm{PaCO}_{2} \\
(\mathrm{kPa})\end{array}$ & Heart rate $(/ \mathrm{min}) \quad \begin{array}{l}\text { Respiratory rate } \\
(/ \mathrm{min})\end{array}$ \\
\hline Mean & $0.97 / 2.42$ & 8.05 & 5.36 & 96 & 21 \\
SD & $0.50 / 1.09$ & 1.52 & 0.88 & 22 & $5 \cdot 11$ \\
\hline
\end{tabular}

$\mathrm{PaO}_{2}$-arterial oxygen tension; $\mathrm{PaCO}_{2}$ - arterial carbon dioxide tension; VAB - visual analogue score for breathlessness. Conversion of SI to traditional units-Blood gas tensions: $1 \mathrm{kPa}=7.5 \mathrm{~mm} \mathrm{Hg}$.

physiological indices to assess the true pharmacological value of oxygen prescribed for the relief of exercise induced dyspnoea.

\section{Methods}

We studied 19 patients (16 male and three female, mean age 65 , range 55-74 years), all of whom had severe obstructive airways disease with variable hypoxaemia and whose principal complaint was shortness of breath on exertion (table 1). At the time of testing all patients were in a stable period free of infection or change in exercise capacity. Before the start of the first of three exercise periods blood was drawn for arterial blood gas analysis and spirometry was performed. The patient was connected to a strain gauge and ECG monitor for respiratory rate and heart rate measurement. A transcutaneous electrode (Radiometer, Copenhagen Ltd) for continuous transcutaneous oxygen $\left(\mathrm{tcPO}_{2}\right)$ estimation was attached to the patient's right shoulder. During a 20 minute period before the start of testing baseline readings of all three physiological indices were made and a visual analogue scale was used for the estimation of breathlessness at rest. Three identical exercises were performed. In each case the patient was asked to step on to and off a secure stool (height $31 \mathrm{~cm}$ ) with the assistance of a hand rail in a manner analogous to climbing stairs. When further exercise was thought by patients to be impossible because of dyspnoea (VAB max) they sat down. Immediately, an MC mask delivering $67 \%$ oxygen or air at the same flow rate (placebo) or no mask at all was placed over the patient's face and recovery was permitted while measurements of the physiological indices were measured and visual analogue scores were determined at half minute intervals until the patient indicated that his breathing was back to "normal" or baseline levels. The mask (if any) was removed, and during a further 20 minute rest measurements continued at one minute intervals for five minutes and thereafter at five minute intervals for the remainder of the 20 minute recovery period. Exercise was then repeated in an identical manner until all three recovery periods were complete. Oxygen, air, and "no mask" were applied in random order according to random number tables.
This entire procedure was repeated in seven patients after an interval of one week to 12 months from the initial test to assess reproducibility. These patients were selected as representing "good" responders, "fair" responders, and non-responders to oxygen assisted recovery and the order of gas administration was randomised afresh.

The data were not normally distributed and statistical analysis was carried out with a non-parametric multiple comparison test ${ }^{9}$ to establish any relationship between speed of recovery to baseline levels of heart rate, respiratory rate, and visual analogue breathlessness in air and oxygen and the "no mask" condition. Duplicate data are shown graph- o ically for the seven patients tested twice to assess

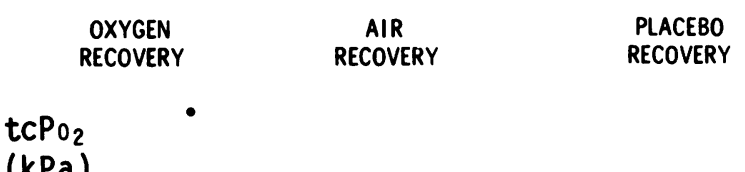

\section{( $\mathrm{KPa})$}
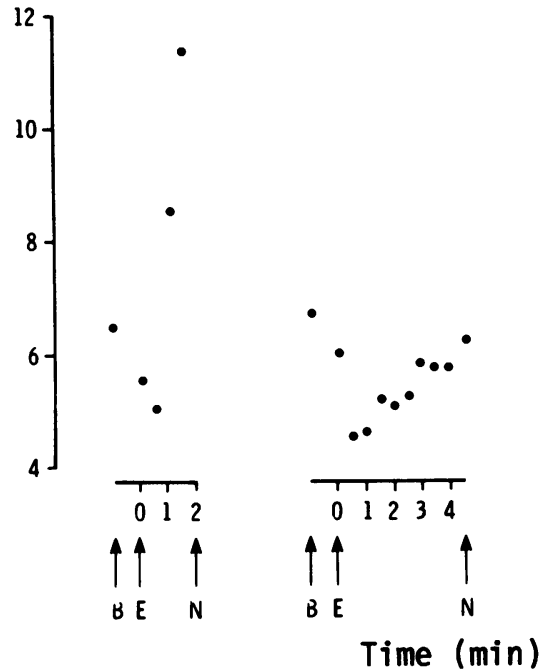

Fig 1 Typical oxygen tension values derived from transcutaneous electrode $\left(t c \mathrm{PO}_{2}\right)$ for one patient showing a good response. Note that the recovery time for breathlessness as assessed on the visual analogue scale is halved during breathing of oxygen. $B$-baseline; $E$-maximum exercise; $N$-claimed normal. 
Short burst oxygen treatment for breathlessness in chronic obstructive airways disease

Patient 1

Patient 2

Patient 3

Patient 4

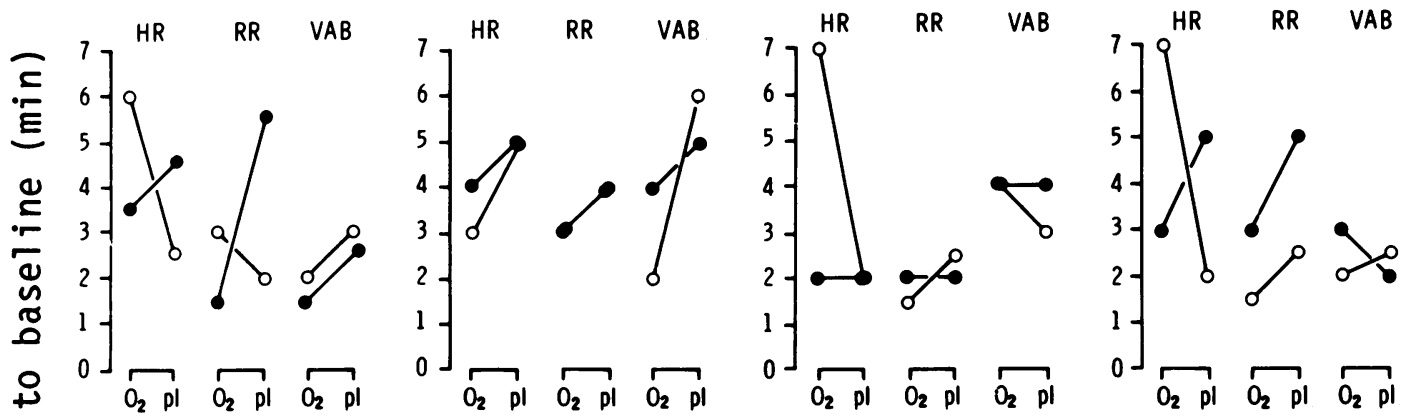

\& Patient $5 \quad$ Patient 6

Patient 7

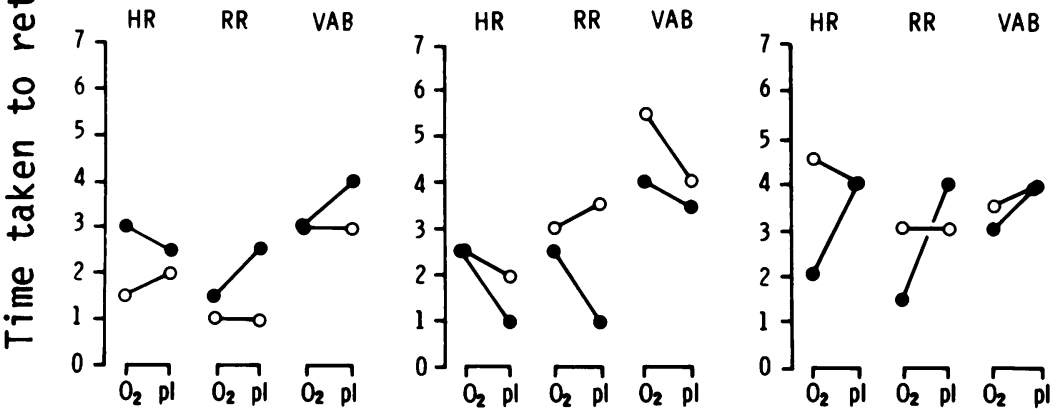

Fig 2 Reproducibility of recovery period for each of three indices ( $H R$-heart rate; $R$-respiratory rate;

$V A B$-breathlessness (assessed on visual analogue scale) in seven patients with oxygen $\left(\mathrm{O}_{2}\right)$ and placebo (pl) during first (O) and second (O) studies.

reproducibility of performance. An attempt was made to analyse these data statistically.

The overall variability in response (heart rate, respiratory rate, and breathlessness) was considered to consist of two components - the variability due to differences in response between individuals and variability due to different responses by the same individual on separate occasions. The relative size of these two values was taken as an index of reproducibility.

\section{Results}

Table 1 shows baseline values of spirometric indices, blood gas tensions, heart rate, respiratory rate, and breathlessness at rest as assessed by the visual analogue scale. Figure 1 shows $\mathrm{tcPO}_{2}$ before, during and after exercise in one patient under three different conditions of recovery. The intervals between measurements were the same in all studies.

Table 2 Time (minutes) taken for heart rate, respiratory rate, and visual analogue breathlessness score (VAB) to return to baseline under different conditions

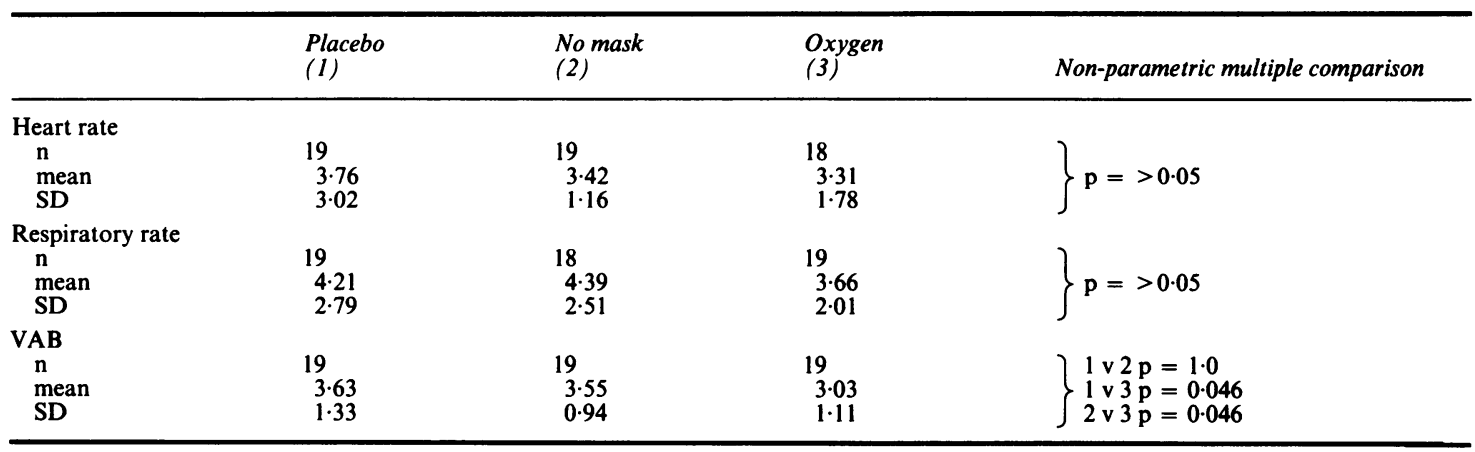


Table 3 Mean sum of squares due to interaction of patients and conditions and due to differences within patients in their responses under the three conditions (variance ratio of greater than about 2 indicates a significant $(p<0.05)$ interaction)

\begin{tabular}{llll}
\hline & Interaction patients $\times$ conditions & Within patients & Variance ratio \\
\hline Visual analogue score of breathlessness & 1.83 & 0.37 & 5.00 \\
Respiratory rate & 4.16 & 2.69 & 1.55 \\
Heart rate & 3.42 & 1.32 & 2.59 \\
\hline
\end{tabular}

The times taken for heart rate, respiratory rate, and breathlessness to return to baseline after the exercises are shown in table 2. Duplicate data for patients tested on two separate occasions to assess reproducibility are shown in figure 2 , with the statistical analysis in table 3. Randomisation of the order of gas administration is shown in table 4 .

The results indicate that mean recovery time with oxygen as judged by visual analogue scale was significantly shorter than when placebo or air recovery was used $(p<0.05)$. Eleven of the 19 patients recovered from exercise faster with oxygen than with placebo or air. The rates of return to baseline levels of respiratory rate and heart rate were not significantly affected by the gas inhaled (table 2). Transcutaneous oxygen tensions were lower than those obtained by arterial sampling $\left(\mathrm{PaO}_{2}\right)$-mean $\mathrm{tcPO}_{2}$ was 6.33 (SD $1.46) \mathrm{kPa}$ compared with $\mathrm{PaO}_{2} 7.53(1.29) \mathrm{kPa}$. Mean $\mathrm{tcPCO}_{2}$, however, was increased to $6.07(1 \cdot 16) \mathrm{kPa}$ compared with $5.43(0.99) \mathrm{kPa}$ for mean $\mathrm{PaCO}_{2}$, which is characteristic of this instrument. A small under-reading of the absolute values of arterial oxygen tension does not affect our argument and corrections have not been made. The time lag of these instruments in responding to changes in arterial gas tension is well known. Measurements of $\mathrm{tcPO}_{2}$ taken at the start of each exercise period were remarkably constant, suggesting that 20 minutes' complete rest after each recovery time was an adequate margin for arterial oxygen tensions to return to normal. Mean recovery time in terms of the visual analogue scale, however, became progressively longer after successive periods of exercise $(3.05(1.21), 3.52(0.94), 3.63(1.21)$ $\mathrm{min})$, although the mean duration of exercise remained constant $(89(61), 98(81), 93(73)$ seconds), suggesting that there was no "practice" effect on exercise performance. Eleven of the 19 patients recovered faster with oxygen than placebo or with no mask and several of these showed a substantial response. An

Table 4 Randomisation order of no mask and of placebo (air) and oxygen administration

\begin{tabular}{llll}
\hline Order & No mask & Placebo & Oxygen \\
\hline 1 & 7 & 6 & 6 \\
2 & 6 & 6 & 7 \\
3 & 6 & 7 & 6 \\
\hline
\end{tabular}

attempt was made to predict such good responders but there was no relationship between speed of recovery with oxygen and initial arterial oxygen tension at rest $(\mathrm{r}=0.19, \mathrm{p}>0.05)$ or $\mathrm{tcPo}_{2}$ immediately after exercise with no mask $(r=0.46, p>0.05)$. Speed of recovery was defined as the increment or decrement of visual analogue breathlessness during oxygen recovery compared with that during "no mask" recovery. A correlation was shown between speed of recovery and the increment of $\mathrm{tcPO}_{2}(\mathrm{r}=0.79, \mathrm{p}<0.001)$. This is likely to reflect the aforementioned time lag in the transcutaneous response to changes in arterial gas tension in that those patients needing the longest time to recover with oxygen had time to register the highest values of $\mathrm{tcPo}_{2}$.

We examined the question of whether the observed differences between the patients in their comparative responses under the three conditions represented real differences by using the additional data on the seven patients who were retested after an interval of from one week to 12 months from the initial study. The $\overrightarrow{\overrightarrow{0}}$ relevant part of the analysis of variance in the data on 3 the 12 patients tested once was compared with that of the seven patients tested twice (table 3 ). The variation in performance "within patients" (that is, when the two performances of each patient were compared) was significantly smaller than that between patients $\stackrel{\otimes}{x}$ (that is, when the single performances of individual 0 patients were compared), only in the case of visual analogue scores of breathlessness.

Despite the apparent reproducibility of this index, examination of the data shows that the direction of $\frac{}{2}$ change in recovery produced by the breathing of $D$ oxygen was variable. In three of the seven patients there was consistent improvement in recovery with o oxygen in both studies; in one patient there was consistent deterioration and in the remaining three 0 patients there was no consistent direction of change in $\omega$ recovery in response to oxygen.

\section{Discussion}

Previous studies, using portable oxygen during exer- $T$ cise, have shown exercise capacity and breathlessness $\frac{\vec{D}}{\mathbb{D}}$ not to be closely related. Breathlessness during exer- $\stackrel{?}{\square}$ cise as measured by a visual analogue scale is $\stackrel{\mathbb{Q}}{2}$ improved by breathing oxygen to a greater extent than is the distance walked. ${ }^{5}$ The findings of this $O$ 
study complement the earlier work. Oxygen aided the recovery of patients after exercise in 11 of our 19 patients. The type of exercise was severe but, even so, the rapid rise in visual analogue scores of breathlessness from a low value after 20 minutes' rest to near the maximum value of 100 within a 78 second period of exercise simply illustrates the rapid onset of dyspnoea so often described by these patients. The shortening of the period required for breathlessness to return to baseline during oxygen breathing amounted to only about 30 seconds in 3.5 minutes (table 2) but this is probably recognised as useful by the patient. This is, of course, a mean value and some individuals had a greater response. Placebo effects were small. The results give little justification for the frequent short term use of oxygen in the home but underline the need to select patients with at least a halving of recovery time while having oxygen and thus a response making treatment worthwhile. We could find no positive relationship between initial $\mathrm{PaO}_{2}$, the fall of $\mathrm{tcPO}_{2}$ during exercise, and the $\mathrm{tcPo}_{2}$ increment during exercise and the response.

The progressive lengthening of recovery time after exercise was an interesting feature of this study. As the duration of exercise remained constant for each exercise period the recovery time between studies was clearly adequate. The randomisation of the order of gas administration was balanced (table 4) and was therefore not responsible for the progressive lengthening of recovery time (as judged by visual analogue score) after successive exercise periods. Possibly some unidentified influence, possibly metabolic, on breathlessness resulted in this anomaly.

Statistical analysis indicated that, of the indices tested, only recovery judged by the visual analogue score of breathlessness might be able to provide a reproducible index of response to oxygen treatment administered in this manner. Despite this, the raw data obtained from the seven patients showed that only four of the seven had a qualitatively similar change on the two occasions. Clearly the change in visual analogue score of breathlessness during one test cannot accurately predict those patients who might receive benefit from short burst oxygen treatment after exercise. Individual patients showing a favourable response should be tested on several separate occasions to establish reproducibility. Probably the number of patients with obstructive lung disease who will have reproducible substantial benefit from short burst oxygen will be very small. If this is confirmed the prescription of oxygen cylinders for occasional use that is now so widespread should largely cease.

Dr T W Evans was a research fellow of the Trent Regional Health Authority Locally Organised Research Committee.

\section{References}

1 McGavin CR, Gupta SP, McHardy GJR. Twelve minute walking test for assessing disability in chronic bronchitis. $\mathrm{Br}$ Med J 1976;i:822-3.

2 Leitch AG, Hopkin JM, Ellis DA, Merchant S, McHardy GJR. The effect of aerosol ipratropium bromide and salbutamol on exercise tolerance in chronic bronchitis. Thorax 1978;33:711-3.

3 Jones MM, Harvey JE, Tattersfield AE. How patients use domiciliary oxygen. $\mathrm{Br} \mathrm{Med} J$ 1978;ii:1397-400.

4 Howard P, Middleton HC. Domiciliary oxygen. $\mathrm{Br}$ Med J 1978;ii:432.

5 Waterhouse JC, Howard P. Breathlessness and portable oxygen in chronic obstructive airways disease. Thorax 1983;38:302-6.

6 Woodcock AA, Gross ER, Geddes DM. Oxygen relieves breathlessness in pink puffers. Lancet 1981;i:907-9.

7 Bond D, Lader M. The use of analogue scales in rating subjective feelings. Br J Med Psychol 1974;47:211-8.

8 Stark RD, Guz A. Dyspnoea. Macclesfield: ICI Pharmaceuticals Division, 1984.

9 Shuster JB, Jones M. Non-parametric multiple comparison procedures. J Am Statist Ass 1974;74:379-82. 